OPEN ACCESS

Edited by:

Andy Mark Borman,

Public Health England,

United Kingdom

Reviewed by:

Alexandre Alanio,

Université Paris Diderot, France

Adilia Warris,

University of Exeter, United Kingdom

${ }^{*}$ Correspondence:

Jean-Pierre Gangneux

jean-pierre.gangneux@univ-rennes1.fr

${ }^{\dagger}$ These authors have contributed equally to this work

Specialty section:

This article was submitted to

Fungal Pathogenesis,

a section of the journal

Frontiers in Cellular and Infection

Microbiology

Received: 03 October 2020 Accepted: 24 December 2020 Published: 18 February 2021

Citation:

Guegan $H$, Prat E, Robert-Gangneux $F$ and Gangneux J-P (2021) Azole Resistance in Aspergillus fumigatus: A

Five-Year Follow Up Experience in a Tertiary Hospital With a Special Focus on Cystic Fibrosis. Front. Cell. Infect. Microbiol. 10:613774. doi: 10.3389/fcimb.2020.613774

\section{Azole Resistance in Aspergillus fumigatus: A Five-Year Follow Up Experience in a Tertiary Hospital With a Special Focus on Cystic Fibrosis}

\author{
Hélène Guegan ${ }^{1,2 \dagger}$, Emilie Prat ${ }^{1 \dagger}$, Florence Robert-Gangneux ${ }^{1,2}$ \\ and Jean-Pierre Gangneux ${ }^{1,2^{*}}$ \\ 1 Laboratoire de Parasitologie-Mycologie, Centre Hospitalier Universitaire de Rennes, Rennes, France, 2 Univ Rennes, CHU, \\ Inserm, Irset (Institut de Recherche en santé, environnement et travail) - UMR_S 1085, Rennes, France
}

Azole-resistant Aspergillus fumigatus (ARAf) has emerged worldwide during the last decades. Drug pressure after long term treatments of chronically infected patients and the propagation of environmental clones selected under the pressure of imidazoles fungicides used in agriculture and farming both account for this emergence. The objectives of this study were to determine the rate of azole resistance in Aspergillus fumigatus during a 5-year period, taking into account (i) differences between underlying diseases of the patients treated, (ii) cross-resistance between azoles, and (iii) focusing on the 5-year evolution of our center's cystic fibrosis cohort. Overall, the rates of voriconazole (VRC)-resistant and itraconazole (ITC)-resistant $A$. fumigatus isolates were 4.1\% (38/927) and $14.5 \%$ (95/656), respectively, corresponding to 21/426 (4.9\%) and 44/308 (14.3\%) patients, respectively. Regarding cross-resistance, among VRC-R isolates tested for ITC, nearly all were R (20/21;95\%), compared to only $27 \%$ (20/74) of VRC-R among ITC-R isolates. The level of azole resistance remained somewhat stable over years but greatly varied according to the azole drug, patient origin, and clinical setting. Whereas azole resistance during invasive aspergillosis was very scarce, patients with cystic fibrosis were infected with multiple strains and presented the highest rate of resistance: 5\% (27/539) isolates were VRC-R and 17.9\% (78/436) were ITC-R. These results underline that the interpretation of the azole resistance level in Aspergilllus fumigatus in a routine setting may consider the huge variability depending on the azole drug, the clinical setting, the patient background and the type of infection.

Keywords: azole resistance, cyp51A, Aspergillus fumigatus, cystic fibrosis, invasive aspergillosis, hematology, intensive care unit 


\section{INTRODUCTION}

Aspergillus fumigatus, a ubiquitously distributed opportunistic pathogen, is the leading agent of aspergillosis, ranking first among fungal killers. In profoundly immunocompromised patients, the clinical picture rapidly evolves towards an acute angio-invasive form or invasive aspergillosis (IA) that accounts for one of the major severe invasive fungal diseases (Ullmann et al., 2018). In the immunocompetent individual, the infection is generally limited to cavitating, chronically evolving forms (such as aspergilloma), chronic fibrotic or immuno-allergic forms (allergic bronchopulmonary aspergillosis and severe asthma with Aspergillus sensitization) (Denning et al., 2016). During cystic fibrosis, abnormally viscous bronchial secretions prevent mucociliary clearance and promote the trapping and the proliferation of inhaled bacteria and fungal spores (Prigitano et al., 2017). A. fumigatus is the most frequent filamentous fungus colonizing the airways of patients with cystic fibrosis (CF) followed by Scedosporium sp. (Pihet et al., 2009; Felton and Simmonds, 2014; Hamprecht et al., 2018). Recent publications underlined that patients with Aspergillus in the airway have greater abnormalities on CT imaging, particularly in children, at the time of infection and in the following years, as shown in longitudinal studies (Breuer et al., 2019).

Voriconazole (VRC), isavuconazole (ISA), itraconazole (ITC), and posaconazole (POS) are four triazole antifungals recommended as first-line drugs in the treatment or prophylaxis of aspergillosis. They inhibit the lanosterol 14- $\alpha$ demethylase enzyme (Cyp51A) encoded by the cyp51A gene, thereby inhibiting the ergosterol synthesis. Voriconazole and isavuconazole are highly recommended for the treatment of IA (Ullmann et al., 2018). Posaconazole shows a very wide spectrum of activity and its primary clinical indications are i) salvage therapy for patients with IA and ii) prophylaxis in patients with neutropenia and hematopoietic cell transplantation (Ullmann et al., 2018). It has also been reported as an alternative treatment for ABPA in CF patients (Periselneris et al., 2019). Itraconazole is the oldest but still robust triazole drug used in chronic and immuno-allergic aspergillosis and allows minimizing the use of corticosteroids (Denning et al., 2016). Azole resistance in A. fumigatus isolates is increasingly reported with variable prevalence in the six continents (Chowdhary et al., 2013; Meis et al., 2016; Wiederhold and Verweij, 2020). Two main origins of the emergence of azole resistant A. fumigatus (ARAf) are recognized: (i) long-term use of triazoles in patients with chronic Aspergillus infections and (ii) increased use of agricultural fungicides against plant-pathogenic moulds, such as Fusarium, Mycosphaerella and A. flavus, with cross-activity against $A$. fumigatus (Meis et al., 2016).

A global rate of resistance of $A$. fumigatus is however a metric with little significance, especially if we do not take into account variability between patients and drugs. During CF, particularlyhigh rates of ARA $f$ have been reported (Stevens et al., 2003; Mortensen et al., 2011; Burgel et al., 2012; Morio et al., 2012; Meis et al., 2016; Prigitano et al., 2017; Risum et al., 2020; Wiederhold and Verweij, 2020) while azoles are recommended in the guidelines for the management of allergic bronchopulmonary aspergillosis (ABPA) (Stevens et al., 2003).

The aim of this prospective study was to estimate the frequency of azole resistance in A. fumigatus isolated from patients in our tertiary care University hospital, over a 5-year period. In particular, we analyzed the prevalence of ARAf according to patient background and azole drug, the cross resistance between azoles, and the characteristics of the longitudinal carriage of Aspergillus in CF patients, and more particularly of ARAf.

\section{METHODS}

\section{Patients}

Data of antifungal susceptibility testing performed on $A$. fumigatus clinical isolates as a part of the routine lab procedure between January 2015 and December 2019 were retrospectively collected. A total of 929 A. fumigatus isolates from 426 patients were tested for susceptibility to at least one azole. Among them, 595 isolates were obtained from sputum samples or throat swabs from $123 \mathrm{CF}$ patients included in the cohorts of adult $(\mathrm{N}=87)$ and pediatric $(\mathrm{N}=36)$ "Centres de Ressources et de Compétences de la Mucoviscidose" at Rennes University Hospital (France). The remaining strains were isolated from patients hospitalized in ICU $(\mathrm{N}=57)$, Pulmonology $(N=159)$, Hematology $(N=13)$ or other clinical wards $(\mathrm{N}=105)$, and used for comparison to isolates from CF patients.

\section{Mycological Examination}

(i) Culture and identification. All isolates of Aspergillus fumigatus were obtained after a culture of respiratory samples, using two Sabouraud dextrose agar slants supplemented with $0.5 \%$ chloramphenicol incubated at $30^{\circ} \mathrm{C}$ and $37^{\circ} \mathrm{C}$ for up to 7 days. Depending on the date of isolation, identification was performed using macroscopic and microscopic examinations of cultures, combined to MALDI-TOF mass spectrometry (MALDI Biotyper, Brucker France, Marne-la-Vallée) or molecular sequencing since 2018.

(ii) In vitro susceptibility to azoles. Itraconazole (ITC), voriconazole (VRC) and posaconazole (POS) minimum inhibitory concentrations (MICs) were determined using Etest $^{\circledR}$ strips and RPMI medium (Biomérieux, MarcyL'Etoile, France) according to the manufacturer recommendations. MICs were determined following a $48 \mathrm{~h}$ incubation at $37^{\circ} \mathrm{C}$. Strains with MIC $\geq 2 \mathrm{mg} / \mathrm{L}$ for ITC and VRC, and $\geq 0.25 \mathrm{mg} / \mathrm{L}$ for POS, were considered resistant (R), according to recent EUCAST breakpoints for fungi (European Committee on Antimicrobial Susceptibility Testing (EUCAST), 2020). Of note, isolates with MICs at 2 $\mathrm{mg} / \mathrm{L}$ for ITC and VRC and $0.25 \mathrm{mg} / \mathrm{L}$ for POS (graded in ATU group, Area of Technical Uncertainty) remain suitable drugs for treatment depending on the clinical setting, 
following latest EUCAST recommendations (European Committee on Antimicrobial Susceptibility Testing (EUCAST), 2020). All other MICs classified isolates as susceptible (S), and therapeutic advice was given with the recommendation to use the tested drug.

(iii) Molecular characterization of cyp51A gene. While not performed routinely, the promoter and the whole cyp51A gene were sequenced in both strands from A. fumigatus isolates in some patients with repeated high MICs and/or clinical failure, using five sets of primers: PA5 and PA7 (Mellado et al., 2001), AF306F and AF855R, AF766F and AF1330R, AF1179F and AF1709R, and AF1426F and AF2025R (Alanio et al., 2011).

The PCR mixture contained $5 \mu \mathrm{l}$ of DNA extract and $20 \mu \mathrm{l}$ of a mix composed of $0.625 \mathrm{U}$ of GoTaq ${ }^{\circledR}$ Hot Start Polymerase (Promega, Charbonnières-les-Bains), 1x Colorless GoTaq ${ }^{\circledR}$ Flexi Buffer (Promega), $2 \mathrm{mM}$ of $\mathrm{MgCl} 2$ (Promega), $0.8 \mathrm{mM}$ of dNTP mix (Eurobio, Les Ulis), and $0.2 \mu \mathrm{M}$ of each primer. The amplification program consisted of $5 \mathrm{~min}$ at $94^{\circ} \mathrm{C}, 30$ cycles of $30 \mathrm{~s}$ at $94^{\circ} \mathrm{C}, 30 \mathrm{~s}$ at $58^{\circ} \mathrm{C}$, and $1 \mathrm{~min}$ at $72^{\circ} \mathrm{C}$, followed by a final step of $10 \mathrm{~min}$ at $72^{\circ} \mathrm{C}$. Sequences of resistant strains were compared to the wild-type A. fumigatus sequence CM 237 (GenBank accession number AF338659), at http://blast.ncbi. nlm.nih.gov/Blast.cgi.

\section{Ethics and Statistics}

According to the French Public Health Laws (Code de la Sante Publique, 2017), protocols of this type do not require approval from an ethics committee and are exempt from the requirement of formal informed consent. MIC values were expressed as median and interquartile range. Data analysis was performed using the GraphPad PRISM ${ }^{\circledR}$ v.5.02 software. For comparison of proportion, the Fisher's test was used. A p-value of 0.05 was considered statistically significant.

\section{RESULTS}

\section{Global Prevalence of Resistance in Azoles}

Before 2015, A. fumigatus isolated in our center were considered as a priori susceptible to azoles and resistance monitoring with MICs was only performed in case of treatment failure. The growing number of treatment failures stressed the need to perform a systematic MIC determination to VRC and/or ITC, for each A. fumigatus isolated from $01 / 2015$ to $01 / 2020$. A total of 927 and 656 strains were evaluated for VRC and ITC susceptibility, respectively. Susceptibility to POS was tested in case of MIC $\geq 2 \mathrm{mg} / \mathrm{L}$ for VRC and ITC, or when the patient had a history of ARAf carriage.

From 2015 to 2019, the rates of VRC-resistant and ITCresistant A. fumigatus isolates were 4.1\% (38/927) and 14.5\% (95/ $656)$, respectively; they were recovered from $21 / 426(4.9 \%)$ and $44 / 308$ (14.3\%) patients, respectively. During the last 4 years with an exhaustive dataset of VRC MIC determination (2016-2019), the rate of VRC-resistant $A$. fumigatus remained stable, with $3.8 \%(8 / 183), 4.8 \%(10 / 209), 3.0 \%(6 / 201)$, and $3.6 \%(8 / 223)$ (ns), with an overall median MIC at $4[2 ; 8] \mathrm{mg} / \mathrm{L}$ (Figure 1). An exhaustive dataset was obtained during the last 3 years for ITC and showed consistent rate at $9.7 \%(20 / 206)$ in 2017, increasing to $16.8 \%(34 / 201)$, and $17 \%(30 / 176)$ in 2018 and 2019 , respectively (ns). The median MIC of ITC-resistant isolates was 14 [3; 32] $\mathrm{mg} / \mathrm{L}$. Among these resistant strains, 20/95 (21\%) isolates and 10/38 (26.3\%) had ITC MICs and VRC

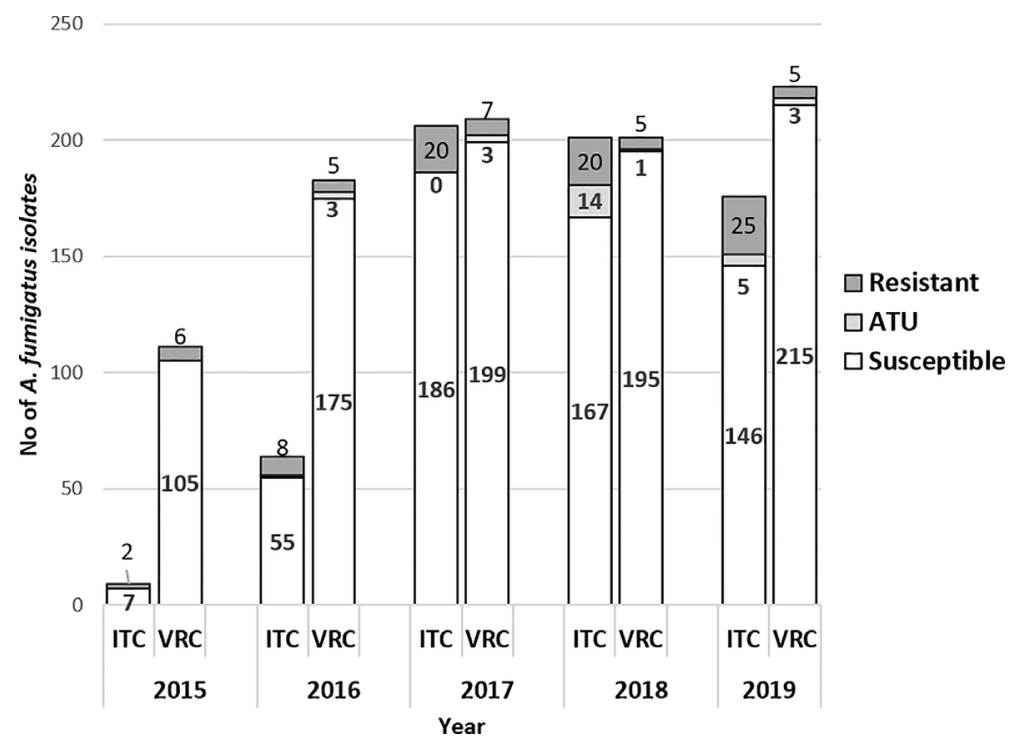

FIGURE 1 | Number of $A$. fumigatus strains susceptible, ATU and resistant to ITC and VRC per year (2015-2019, N = 929) $($ VRC, $n=927)$; (ITC, $n=656)$. White bars indicate susceptible isolates; dark grey bars indicate resistant isolates with MIC $>2 \mathrm{mg} / \mathrm{L}$ and light grey bars represent isolates resistant isolates in ATU group (MIC = 2 mg/L). ITC, itraconazole; VRC, voriconazole; ATU, Area of Technical Uncertainty. 
MICs in the ATU range, respectively, corresponding to 20/656 (3.0\%) and 10/927 (1.1\%) of all tested isolates for ITC and VRC, respectively (Figure 1).

\section{Prevalence of Azole Resistance According to the Clinical Setting Patients From the Cystic Fibrosis Cohort}

CF patients are managed in special units. They are sometimes hospitalized in other Pulmonology units, but we have reclassified them as "CF patients" because this cohort of patients is followed individually. A total of 595 A. fumigatus isolates were collected from respiratory samples of $123 \mathrm{CF}$ patients and screened for VRC and/or ITC and/or POS in vitro susceptibility, of whom 34 patients presented with at least one isolate resistant to one drug. The rate of isolates resistant to at least one azole reached 90/595 (15.1\%) over the period 2015-2019, among which three isolates were only POS-R. Regarding VRC, the global frequency of resistant A. fumigatus strains from 2016 to 2019 was 5\% (27/ 539), recovered from 13 out of 119 patients (10.9\%). The rate of VRC-R isolates was relatively steady from 2016 to 2019 (Figure 2). Over the same period (2016-2019), the rate of ITC-R isolates was higher than that of VRC-R, with 78/436 (17.9\%) resistant isolates, recovered from $29 / 111$ (26.1\%) patients. A tendency to increase was noticed, with rates of ARAf detection from 14 to $24 \%$ from 2017 to 2019 , respectively.

\section{Pulmonology}

A total of 159 isolates from 143 patients hospitalized in pulmonology, excluding CF patients, were screened for azole susceptibility between 2015 and 2019. Only four isolates from four patients were resistant to ITC (including one ATU isolate), of which one was also resistant to VRC (Table 1). Another isolate was ATU to VRC only.

\section{Intensive Care Units}

Fifty-seven isolates from 50 patients were screened for azole resistance from 2015 to 2019. Six patients harbored an ITC-R isolate (of which 4 were ATU) and two isolates from one patient were VRC-R, none of them with azole cross resistance (Table 1). None of these patients were CF. A few patients with

TABLE 1 | Itraconazole (ITC) and voriconazole (VRC) resistance of $A$. fumigatus isolates from patients from pulmonology (excluding CF), intensive care unit, and other units.

\begin{tabular}{|c|c|c|c|c|c|c|}
\hline \multirow[t]{3}{*}{ Year } & \multicolumn{6}{|c|}{ Number of $A$. fumigatus isolates resistant to antifungals* (n/N) } \\
\hline & \multicolumn{2}{|c|}{ Pulmonology } & \multicolumn{2}{|c|}{ ICU } & \multicolumn{2}{|c|}{ Other } \\
\hline & ITC & VRC & ITC & VRC & ITC & VRC \\
\hline 2015 & $0 / 1$ & $0 / 30$ & nd & $0 / 9$ & $0 / 4$ & $1 / 18$ \\
\hline 2016 & $0 / 2$ & $0 / 21$ & $0 / 2$ & $0 / 6$ & $0 / 5$ & $0 / 21$ \\
\hline 2017 & $0 / 35$ & 0/35 & $1 / 9$ & $0 / 10$ & $0 / 25$ & $0 / 27$ \\
\hline 2018 & $4 / 31$ & $1 / 31$ & $4 / 15$ & $0 / 15$ & $1 / 15$ & $0 / 15$ \\
\hline 2019 & 0/35 & $1 / 42$ & $1 / 11$ & $2 / 17$ & $4 / 26$ & $1 / 37$ \\
\hline Total & $4^{1 / 104}$ & $2^{2} / 159$ & $6^{3} / 37$ & $2 / 57$ & $5^{4} / 75$ & $2^{2} / 118^{\$}$ \\
\hline$\%$ & $3.8 \%$ & $1.3 \%$ & $16.2 \%$ & $3.5 \%$ & $6.6 \%$ & $1.7 \%$ \\
\hline
\end{tabular}

ICU, intensive care unit; ITC, itraconazole; VRC, voriconazole; nd, not determined * $M I C \geq 2 \mathrm{mg} / \mathrm{L}$.

${ }^{\$}$ Among them, 13 isolates were from hematology patients; 0/13 were resistant.

${ }^{1}$ Among them, $1 / 4$ was ATU.

${ }^{2}$ Among them, $1 / 2$ was ATU.

${ }^{3}$ Among them, 4/6 were ATU.

${ }^{4}$ Among them, $1 / 5$ was ATU.

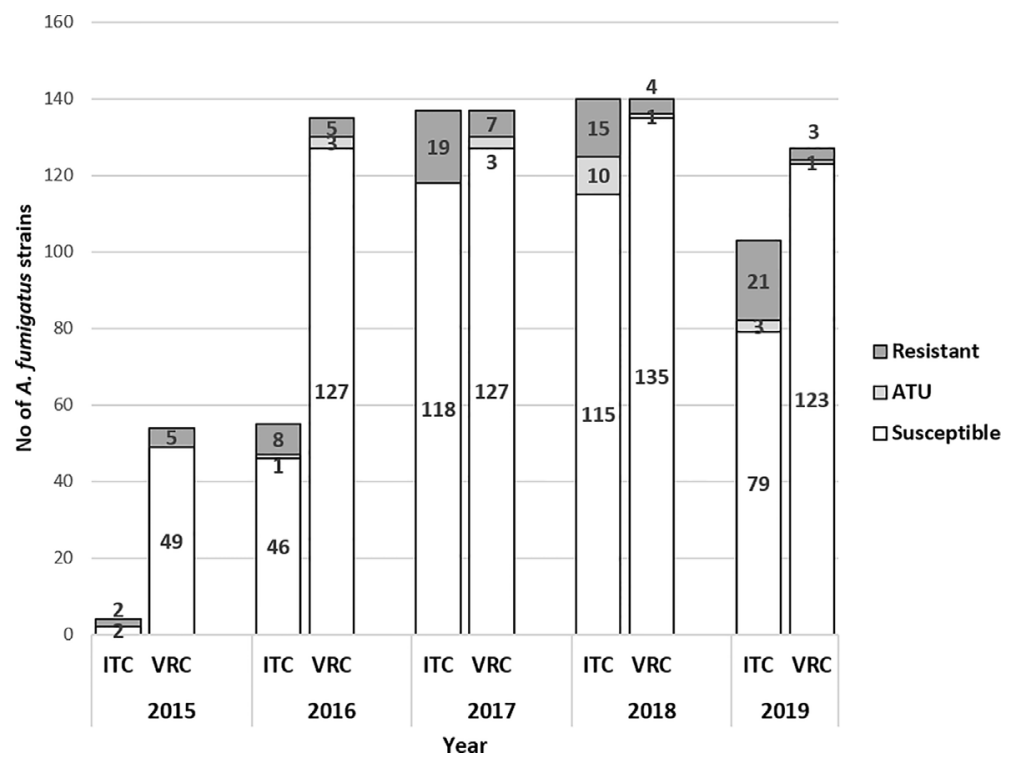

FIGURE 2 | Number of $A$. fumigatus strains susceptible, ATU and resistant to itraconazole (ITC) and voriconazole (VRC) per year in CF patients (2015-2019, N = 595 (VRC, $n=593)$; (ITC, $n=440)$. White bars indicate susceptible isolates; dark grey bars indicate resistant isolates with MIC $>2 \mathrm{mg} / \mathrm{L}$ and light grey bars represent isolates resistant isolates in ATU group ( $\mathrm{MIC}=2 \mathrm{mg} / \mathrm{L})$. ITC, itraconazole; VRC, voriconazole; ATU, Area of Technical Uncertainty. 
hematological malignancies have been occasionally hospitalized in ICU. We keep them as "ICU patients" as the specific patient management in ICU (mechanical ventilation for example) is different and can contribute to modify the epidemiology.

TABLE 2 | Prevalence of azole resistance in A. fumigatus isolates tested simultaneously for the three azole antifungals $(N=56)$.

\begin{tabular}{lcccc}
\hline Susceptibility profile & \multicolumn{3}{c}{ Azole antifungal } & \multirow{2}{*}{ Number } \\
\cline { 2 - 4 } & ITC & VRC & POS & \\
\hline No azole R & S & S & S & 20 \\
R to 1 azole & $\mathbf{R}$ & S & S & 2 \\
& S & R & S & 1 \\
R to 2 azoles & S & S & R & 3 \\
& R & S & $\mathbf{R}$ & 14 \\
R to 3 azoles & S & $\mathbf{R}$ & $\mathbf{R}$ & 1 \\
& $\mathbf{R}$ & $\mathbf{R}$ & S & 1 \\
& $\mathbf{R}$ & $\mathbf{R}$ & $\mathbf{R}$ & 14
\end{tabular}

$R$, resistance; S, susceptibility.

\section{Other Wards}

Cultural analysis in Hematology patients remains rare for the diagnosis of IA compared to galactomannan determination in the serum used for screening in high-risk patients. Only 13 A. fumigatus were isolated and tested in patients with hematological malignancies since 2015 and no azole resistance was detected. These patients are grouped with patients from other units in Table 1. In patients with miscellaneous clinical backgrounds (other units), five were infected with an ITC-R strain (coresistant to VRC in one case), of which one was an ATU isolate.

\section{Cross-Resistance Between Azoles}

We then analyzed whether there was cross-resistance between the main azole antifungals used routinely, in order to help for the decision of doctors excluding ATU isolates. Among the $83 \mathrm{~A}$. fumigatus strains with high MIC for ITC and/or VRC (> $2 \mathrm{mg} / \mathrm{L})$, $20(24 \%)$ were resistant to both ITC and VRC. However, the proportions of ITC-R/VRC-S and ITC-S/VRC-R differed greatly. Among VRC-R isolates tested for ITC, nearly all were R to ITC (20/ $21 ; 95 \%)$, while only $27 \%$ (20/74) of ITC-R isolates were VRC-R.

TABLE 3 | Characteristics of isolates obtained from CF patients with at least one resistance to VRC or ITC over the study period (2015-2019).

\begin{tabular}{|c|c|c|c|c|c|c|c|}
\hline No & $\begin{array}{c}\text { No } \\
\text { ofisolates }\end{array}$ & $\begin{array}{c}\text { No of } \\
\text { ITC-Risolates }\end{array}$ & $\begin{array}{l}\text { MIC range of ITC-R } \\
\text { isolates }\end{array}$ & $\begin{array}{l}\text { No of VRC-R } \\
\text { isolates }\end{array}$ & $\begin{array}{c}\text { MIC range of VRC-R } \\
\text { isolates }\end{array}$ & $\begin{array}{c}\text { Mean time between } \\
\text { isolates (days) }\end{array}$ & $\begin{array}{l}\text { Preexposure } \\
\text { to azole }\end{array}$ \\
\hline 1 & 1 & ND & ND & 1 & 4 & NA & ND \\
\hline 2 & 18 & $11^{\star}$ & $2 ;>32$ & $10^{\star \star \#}$ & $2 ;>32$ & 84 & yes \\
\hline 3 & 16 & $4^{*}$ & $2 ;>32$ & 1 & 16 & 101 & yes \\
\hline 4 & 3 & 1 & 2 & 0 & NA & 458 & yes \\
\hline 5 & 6 & 4 & $4 ; 16$ & 0 & NA & 74 & yes \\
\hline 6 & 4 & 1 & 4 & 0 & NA & 86 & no \\
\hline 7 & 14 & 1 & 2 & $1^{\#}$ & 2 & 73 & no \\
\hline 8 & 13 & 1 & 3 & 0 & NA & 104 & yes \\
\hline 9 & 3 & 1 & $>32$ & 1 & $>32$ & 89 & yes \\
\hline 10 & 9 & 1 & 2 & 0 & NA & 185 & no \\
\hline 11 & 3 & 2 & $>32$ & 1 & 3 & 77 & yes \\
\hline 12 & 11 & 1 & 2 & 0 & NA & 135 & no \\
\hline 13 & 1 & 1 & $>32$ & 0 & NA & NA & no \\
\hline 14 & 6 & 1 & 24 & 0 & NA & 115 & ND \\
\hline 15 & 7 & $3^{*}$ & $2 ; 12$ & 0 & NA & 160 & yes \\
\hline 16 & 2 & 1 & 16 & 0 & NA & 730 & ND \\
\hline 17 & 10 & 3 & $3 ;>32$ & $3^{*}$ & $2 ;>32$ & 146 & yes \\
\hline 18 & 2 & 1 & 8 & 0 & NA & 1267 & yes \\
\hline 19 & 3 & 1 & 8 & 0 & NA & 109 & no \\
\hline 20 & 3 & 1 & 16 & 2 & $3 ; 12$ & 119 & no \\
\hline 21 & 13 & 1 & 2 & 0 & AN & 125 & ND \\
\hline 22 & 5 & 1 & 8 & 0 & NA & 195 & yes \\
\hline 23 & 5 & 5 & 8;32 & 1 & 4 & 210 & yes \\
\hline 24 & 14 & 9 & $2 ;>32$ & 0 & NA & 114 & yes \\
\hline 25 & 1 & 1 & 16 & 1 & 3 & 128 & yes \\
\hline 26 & 9 & 5 & $6 ;>32$ & $2^{*}$ & $2 ; 8$ & 169 & yes \\
\hline 27 & 4 & $2^{\star}$ & $2 ; 3$ & 0 & NA & 54 & no \\
\hline 28 & 8 & 0 & & 1 & 2 & 169 & no \\
\hline 29 & 3 & 1 & 2 & 0 & NA & 476 & no \\
\hline 30 & 12 & $4^{*}$ & $2 ; 16$ & 0 & NA & 123 & no \\
\hline 31 & 13 & $9^{*}$ & $2 ;>32$ & $4^{\star \star}$ & $2 ; 3$ & 111 & yes \\
\hline 32 & 12 & 0 & & 1 & 3 & 138 & no \\
\hline 33 & 2 & 1 & 32 & 1 & 32 & 1320 & no \\
\hline 34 & 7 & $1^{*}$ & 2 & 0 & NA & 187 & no \\
\hline
\end{tabular}

*Indicates the presence of 1 ATU among $R$ isolates.

**Indicates the presence of 2 ATU among $R$ isolates.

"ATU differed from the ITC-ATU isolate.

$N A$, not applicable; ND, not determined; MIC, minimal inhibitory concentration (mg/L). 
In our routine setting, POS is only tested in case of azoleresistance history or clinical failure after VRC or ITC treatment. Of the 56 isolates tested simultaneously for ITC, VRC and POS, $20(36 \%)$ were susceptible to all of them (Table 2). A crossresistance was observed in 54\% (30/56) (Table 2). Two isolates had a decreased susceptibility to ITC only (MIC at 2 and $3 \mathrm{mg} / \mathrm{L}$, respectively), and one isolate had an increased MIC for VRC only (at $2 \mathrm{mg} / \mathrm{L}$ ). Among the 31 ITC-R isolates, 28 (90.3\%) were POS$\mathrm{R}$ whereas only 15 isolates (48.4\%) were VRC-R. Overall, 76 isolates benefited from MIC evaluation to POS, of which 37 (48.7\%) were POS-R. Thirty-four of the 37 (91.9\%) isolates POS$\mathrm{R}$ were also resistant to ITC and/or VRC, of which 32 were observed in CF patients.

\section{Phenotypic and Genotypic Characterization of Isolates from CF Patients}

During the five-year follow-up, 34 out of 123 patients (27.6\%) (90/595 isolates, $15.1 \%)$ presented at least once with an ARAf in induced sputum samples. About half of them (18/34) harbored a single ARAf among several Aspergillus isolates (2 to 18 consecutive isolates), of which seven were ATU (Table 3), whereas three patients had a single positive sputum with ARAf and no further positive Aspergillus cultures (patients \#1, \#13, and $\# 25$, Table 3). As seen in Table 3, the range of ITC and VRC MIC for different ARAffrom a same patient was sometimes huge, suggesting that some patients are colonized with a multiplicity of strains over time. The MIC distribution of all isolates collected from patients with at least one ARAf is shown in Figure 3.

As an example of the multiplicity of strains, distribution of MICs of consecutive isolates from four CF patients with $>10$ isolates were recorded over time. Figure $\mathbf{4}$ shows that for a given patient, MIC values fluctuated over time, with cultures of susceptible and resistant phenotypes, underlining the diversity of A. fumigatus strains colonizing the airways of CF patients.

Cyp51A genotyping of ARA $f$ was performed in strains isolated from six CF patients, reflecting the diversity of genetic alterations related to triazole resistance (Table 4). Interestingly, cyp51A polymorphism were found in $4 / 6$ isolates. While the multi-azole resistant isolate harbored the $\mathrm{L} 98 \mathrm{H}$ mutation together with the tandem repeat TR34 (patient 9), the two ITC-R and POS-R ARAf displayed the substitutions $\mathrm{M} 220 \mathrm{~K}$ and G54R respectively (patients 2 and 24). Finally, the combined F46Y, M172V, and E427K amino acid changes were simultaneously found in the ITC-R isolate. Triazole resistance of isolates from patients \#3 and \#26 was not linked to cyp51A polymorphism (wild type genotype) supporting the existence of other biological mechanisms responsible for triazole resistance.

\section{DISCUSSION}

The burden of fungal diseases due to Aspergillus remains high in France, with multiple forms, from chronic or allergic to invasive aspergillosis (Gangneux et al., 2016). Furthermore, the emergence Aspergillus fumigatus isolates resistant to azoles led international experts to recommend the in vitro susceptibility screening of isolates and to shift from azole to amphotericin B as

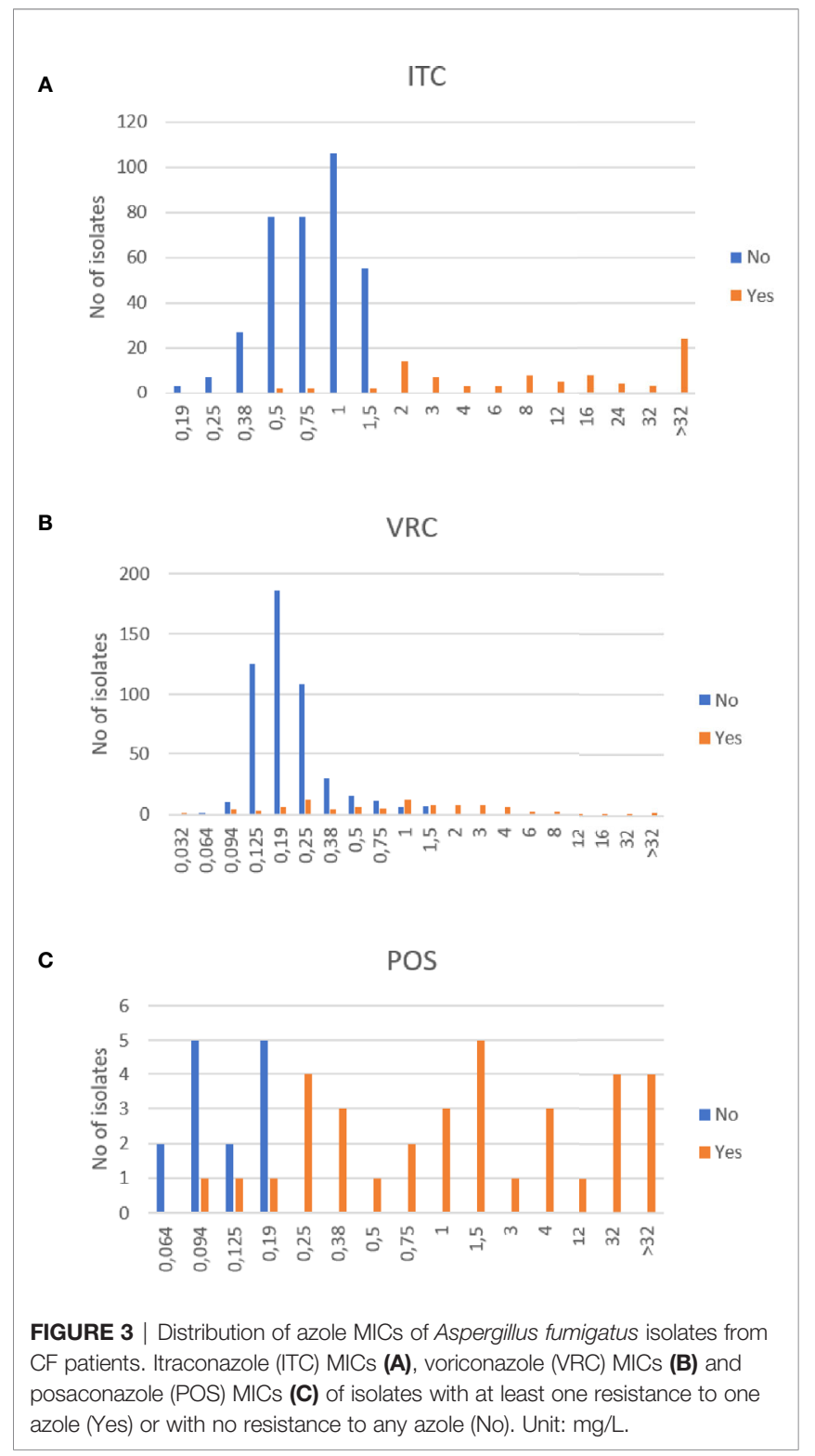

first-line treatment of IA when resistance is higher than $10 \%$ (Verweij et al., 2015; Ullmann et al., 2018).

Here we analyzed the results of in vitro susceptibility of $A$. fumigatus during a 5-year survey in a routine practice. Because the micro-dilution method recommended by EUCAST is difficult to use in routine when numerous isolates are tested per day, we use Etest ${ }^{\circledR}$ strips and RPMI medium in our routine practice as in many hospitals. The global level of resistance of isolates to voriconazole and itraconazole was 4.1 and $14.5 \%$, respectively. This level remained somewhat stable over years. Interestingly, a recent publication from the Netherlands showed that the VRC-resistance frequency was 34\% lower in 2018 than in 2013 ( $\mathrm{p}=0.0001$ ) (Lestrade et al., 2020). However, our results show that the level of resistance greatly varied according to azole drug, patient origin and clinical setting. Chronic respiratory diseases and particularly cystic fibrosis are favorable to ARAf emergence because of recurrent treatment with azoles. Besides, 

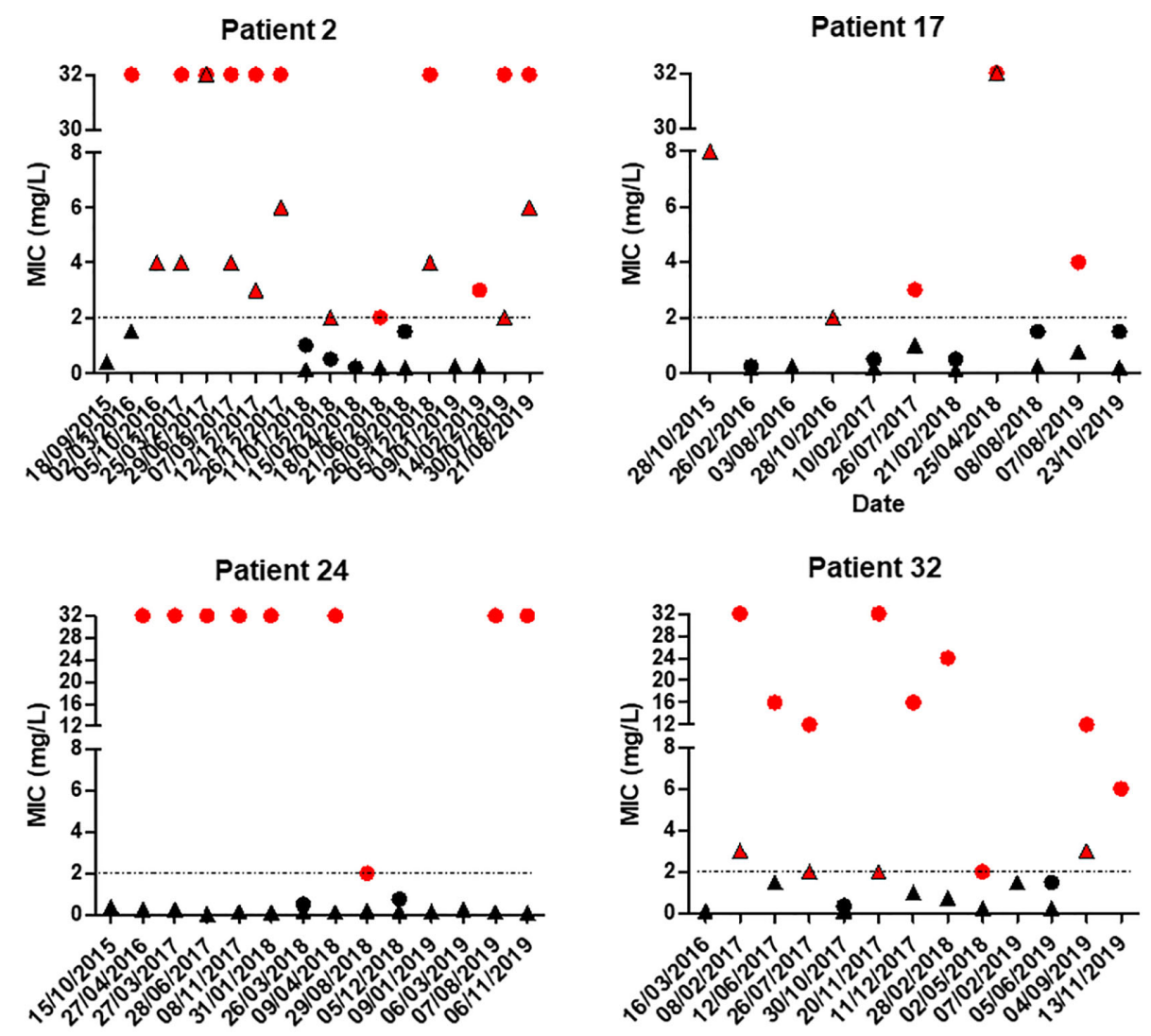

\section{- ITC-S - ITC-R \\ - VRC-S $\triangle$ VRC-R}

FIGURE 4 | Evolution of itraconazole (ITC) and voriconazole (VRC) MIC values of A. fumigatus isolates from four patients in the time. For clarity, isolates with ATU MIC values were gathered with resistant isolates.

TABLE 4 | Profile of azole resistance in CF patients with cyp51A-genotyped ARAf.

\begin{tabular}{lcccl}
\hline No of patient & ITC MIC & VRC MIC & POS MIC & cyp51A mutations \\
\hline 2 & $>32$ & 1.5 & $>32$ & M220K \\
3 & 32 & 16 & 0.094 & none \\
9 & $>32$ & $>32$ & $>32$ & TR34/L98H \\
24 & $>32$ & 0.25 & $>32$ & G54R \\
26 & 32 & 8 & 4 & none \\
30 & 2 & 0.75 & ND & F46Y, M172V, E427K
\end{tabular}

ND, not determined; MIC, minimal inhibitory concentration $(\mathrm{mg} / \mathrm{L})$.

we detected only a very low number of ARAf during invasive pulmonary aspergillosis.

Regarding the management of IPA, a few other French papers from Paris area reported similar low levels of ARAf detection (Alanio et al., 2011; Alanio et al., 2016). At the time of hospitalization in Hematology, none of the patients had an ARA $f$ isolated, probably linked to the short course of chemoprophylaxis with posaconazole. These results comfort us in the use of azoles as first-line curative drugs (VRC and ISA) or prophylaxis (POS).

Regarding CF patients during 2015-2019, we observed a higher frequency of $\operatorname{ARAf}(11 \%)$ than in other French studies. Our center is one of the two tertiary hospitals of the Brittany region which is mainly rural with an intense agricultural and farming activity that could account for high azole resistance rate, and furthermore $16 / 30$ patients with ARAf had a pre-exposure to azoles. In the West of France near Brittany, Morio et al., (2012) reported a $8 \%$ prevalence of azole resistance in 20102011 , while this rate was $6.8 \%$ four years later, mainly due to a genotypic mutation TR34/L98H (Lavergne et al., 2019). In a cohort from Paris, $4.8 \%$ of resistant isolates were detected (Burgel et al., 2012), whereas in a mixed cohort of CF and immunocompromised patients from the North of Paris, only $1.8 \%$ of ARAf were detected (Choukri et al., 2015). European studies showed that $5.3 \%(101 / 2888)$ of $A$. fumigatus isolates were azole-resistant in a prospective multicenter study in Germany (Seufert et al., 2018). The frequency was $7.1 \%$ in the 
Netherlands, with TR34/L98H being the dominant resistance mechanism (Engel et al., 2019). In a bicentric Italian study, no resistance was detected in one center, while the frequency of resistance was $8.2 \%$ in the other (Prigitano et al., 2017). In Denmark, Risum et al. recently reported the occurrence of ARAf strains in $7.3 \%(10 / 137)$ CF patients (Risum et al., 2020).

The issue of cross-resistance remains moderate in our center even if azole exposure is frequent, as VRC remains usable for $73 \%$ of ITC-R isolates, and a triple-resistance to azole was very scarce. Importantly, isolates that were both ITC-R and VRC-R were POS-R except in one case. Thus, we decided to stop POS in vitro susceptibility testing to the benefit of ISA susceptibility testing. Data obtained during this survey incited us to adopt new rules for in vitro testing strategy:

(i) When VRC is considered as the first-line treatment: we perform VRC and ISA susceptibility testing and recommend to switch for liposomal amphotericin $\mathrm{B}$ when VRC-R and ISA-R. Such situation is possible during invasive, allergic and chronic aspergillosis.

(ii) When ITC is considered as the first-line treatment: we perform ITC, VRC and ISA susceptibility testing and first recommend to change for VRC or ISA in case of ITC-R, and to consider liposomal amphotericin B in case of pan-azole-R. Such situation is possible mainly during allergic and chronic aspergillosis.

Finally, during CF, we confirmed the high diversity of Aspergillus isolates. Indeed, CF patients may be colonized with a wide succession of different phenotypes, either susceptible or resistant. This finding could be confirmed by a genotypic characterization of isolates, since it has been described that different genotype, until 5 to 10 , could be isolated in a same patients (Neuvéglise et al., 1997; Amorim et al., 2010). Here again, this result made us change our routine procedure to a systematic antifungal susceptibility testing on various colonies from the same samples, as recommended by international guidelines (Ullmann et al., 2018). And as shown by Lestrade et al. (2020), the monitoring of genotypes may also be combined to in vitro susceptibility determination since they reported that

\section{REFERENCES}

Alanio, A., Sitterle, E., Liance, M., Farrugia, C., Foulet, F., Botterel, F., et al. (2011). Low prevalence of resistance to azoles in Aspergillus fumigatus in a French cohort of patients treated for haematological malignancies. J. Antimicrob. Chemother. 66, 371-374. doi: 10.1093/jac/dkq450

Alanio, A., Denis, B., Hamane, S., Raffoux, E., Peffault de Latour, R., Menotti, J., et al. (2016). Azole Resistance of Aspergillus fumigatus in Immunocompromised Patients with Invasive Aspergillosis. Emerg. Infect. Dis. 22, 157-158. doi: 10.3201/ eid2201.150848

Amorim, A., Guedes-Vaz, L., and Araujo, R. (2010). Susceptibility to five antifungals of Aspergillus fumigatus strains isolated from chronically colonised cystic fibrosis patients receiving azole therapy. Int. J. Antimicrob. Agents 35, 396-399. doi: 10.1016/j.ijantimicag.2009.12.007

Breuer, O., Schultz, A., Garratt, L. W., Turkovic, L., Rosenow, T., Murray, C. P., et al. (2019). Aspergillus Infections and Progression of Structural Lung Disease the mean VRC MIC of TR34/L98H isolates decreased from 8 $\mathrm{mg} / \mathrm{L}$ (2013) to $2 \mathrm{mg} / \mathrm{L}$ (2018) in the Dutch experience.

Overall, this survey underlines a huge variability of azole resistance level depending on the azole drug, the patient origin and the clinical setting, and also variability within a patient. In addition to epidemiological data, this study made us changing our routine procedure of azole in vitro testing. The limits of this field and practical study are its monocentric design, some incomplete data for some patients, and the absence of in vitro data on isavuconazole that was only recently introduced in our centre.

\section{DATA AVAILABILITY STATEMENT}

The raw data supporting the conclusions of this article will be made available by the authors, without undue reservation.

\section{ETHICS STATEMENT}

The studies involving human participants were reviewed and approved by Comité d'éthique de Rennes, France. Written informed consent from the participants' legal guardian/next of kin was not required to participate in this study in accordance with the national legislation and the institutional requirements.

\section{AUTHOR CONTRIBUTIONS}

All authors listed have made a substantial, direct and intellectual contribution to the work, and approved it for publication.

\section{ACKNOWLEDGEMENTS}

We thank Docteur C. Belleguic and E. Deneuville that are responsible for the adult and pediatric "Centres de Ressources et de Compétences de la Mucoviscidose". We thank LGgx for English editing service. in Children with Cystic Fibrosis. Am. J. Respir. Crit. Care Med. 201, 688-696. doi: 10.1164/rccm.201908-1585OC

Burgel, P.-R., Baixench, M.-T., Amsellem, M., Audureau, E., Chapron, J., Kanaan, R., et al. (2012). High Prevalence of Azole-Resistant Aspergillus fumigatus in Adults with Cystic Fibrosis Exposed to Itraconazole. Antimicrob. Agents Chemother. 56, 869-874. doi: 10.1128/AAC.05077-11

Choukri, F., Botterel, F., Sitterlé, E., Bassinet, L., Foulet, F., Guillot, J., et al. (2015). Prospective evaluation of azole resistance in Aspergillus fumigatus clinical isolates in France. Med. Mycol. 53, 593-596. doi: 10.1093/mmy/myv029

Chowdhary, A., Kathuria, S., Xu, J., and Meis, J. F. (2013). Emergence of azole-resistant Aspergillus fumigatus strains due to agricultural azole use creates an increasing threat to human health. PloS Pathog. 9, e1003633. doi: 10.1371/journal.ppat.1003633

Code de la Santé Publique (2017). Décret n²017-884 du 9 mai 2017 modifiant certaines dispositions réglementaires relatives aux recherches impliquant la personne humaine | Legifrance. Article R.1121-1-1. Available at: https://www. legifrance.gouv.fr/eli/decret/2017/5/9/AFSP1706303D/jo/texte. 
Denning, D. W., Cadranel, J., Beigelman-Aubry, C., Ader, F., Chakrabarti, A., Blot, S., et al. (2016). Chronic pulmonary aspergillosis: rationale and clinical guidelines for diagnosis and management. Eur. Respir. J. 47, 45-68. doi: 10.1183/ 13993003.00583-2015

Engel, T. G. P., Slabbers, L., de Jong, C., Melchers, W. J. G., Hagen, F., Verweij, P. E., et al. (2019). Prevalence and diversity of filamentous fungi in the airways of cystic fibrosis patients - A Dutch, multicentre study. J. Cyst. Fibros. 18, 221226. doi: $10.1016 /$ j.jcf.2018.11.012

European Committee on Antimicrobial Susceptibility Testing (EUCAST) (2020). Antifungal Agents-Breakpoint tables for interpretation of MICs, version 10.0. Available at: https://www.eucast.org/fileadmin/src/media/PDFs/EUCAST_ files/AFST/Clinical_breakpoints/AFST_BP_v10.0_200204.pdf (Accessed September 21, 2020).

Felton, I. C., and Simmonds, N. J. (2014). Aspergillus and cystic fibrosis: old disease - new classifications. Curr. Opin. Pulm. Med. 20, 632-638. doi: 10.1097/ MCP.0000000000000106

Gangneux, J.-P., Bougnoux, M.-E., Hennequin, C., Godet, C., Chandenier, J., Denning, D. W., et al. (2016). An estimation of burden of serious fungal infections in France. J. Med. Mycol. 26, 385-390. doi: 10.1016/j.mycmed.2016.11.001

Hamprecht, A., Morio, F., Bader, O., Le Pape, P., Steinmann, J., and Dannaoui, E. (2018). Azole Resistance in Aspergillus fumigatus in Patients with Cystic Fibrosis: A Matter of Concern? Mycopathologia 183, 151-160. doi: 10.1007/ s11046-017-0162-4

Lavergne, R.-A., Morio, F., Danner-Boucher, I., Horeau-Langlard, D., David, V., Hagen, F., et al. (2019). One year prospective survey of azole resistance in Aspergillus fumigatus at a French cystic fibrosis reference centre: prevalence and mechanisms of resistance. J. Antimicrob. Chemother. 74, 1884-1889. doi: 10.1093/jac/dkz144

Lestrade, P. P. A., Buil, J. B., van der Beek, M. T., Kuijper, E. J., van Dijk, K., Kampinga, G. A., et al. (2020). Paradoxal Trends in Azole-Resistant Aspergillus fumigatus in a National Multicenter Surveillance Program, the Netherland2018. Emerg. Infect. Dis. 26 (7), 1447-1455. doi: 10.3201/eid2607.200088

Meis, J. F., Chowdhary, A., Rhodes, J. L., Fisher, M. C., and Verweij, P. E. (2016). Clinical implications of globally emerging azole resistance in Aspergillus fumigatus. Philos. Trans. R. Soc B Biol. Sci. 371, 20150460. doi: 10.1098/ rstb.2015.0460

Mellado, E., Diaz-Guerra, T. M., Cuenca-Estrella, M., and Rodriguez-Tudela, J. L. (2001). Identification of Two Different 14- Sterol Demethylase-Related Genes (cyp51A and cyp51B) in Aspergillus fumigatus and Other Aspergillus species. J. Clin. Microbiol. 39, 2431-2438. doi: 10.1128/JCM.39.,7.2431-2438.2001

Morio, F., Aubin, G. G., Danner-Boucher, I., Haloun, A., Sacchetto, E., GarciaHermoso, D., et al. (2012). High prevalence of triazole resistance in Aspergillus fumigatus, especially mediated by TR/L98H, in a French cohort of patients with cystic fibrosis. J. Antimicrob. Chemother. 67, 1870-1873. doi: 10.1093/jac/dks160

Mortensen, K. L., Jensen, R. H., Johansen, H. K., Skov, M., Pressler, T., Howard, S. J., et al. (2011). Aspergillus Species and Other Molds in Respiratory Samples from Patients with Cystic Fibrosis: a Laboratory-Based Study with Focus on Aspergillus fumigatus Azole Resistance. J. Clin. Microbiol. 49, 2243-2251. doi: 10.1128/JCM.00213-11

Neuvéglise, C., Sarfati, J., Debeaupuis, J. P., Vu Thien, H., Just, J., Tournier, G., et al. (1997). Longitudinal study of Aspergillus fumigatus strains isolated from cystic fibrosis patients. Eur. J. Clin. Microbiol. Infect. Dis. 16, 747-750. doi: $10.1007 /$ BF01709257

Periselneris, J., Nwankwo, L., Schelenz, S., Shah, A., and Armstrong-James, D. (2019). Posaconazole for the treatment of allergic bronchopulmonary aspergillosis in patients with cystic fibrosis. J. Antimicrob. Chemother. 74, 1701-1703. doi: 10.1093/jac/dkz075

Pihet, M., Carrere, J., Cimon, B., Chabasse, D., Delhaes, L., Symoens, F., et al. (2009). Occurrence and relevance of filamentous fungi in respiratory secretions of patients with cystic fibrosis-a review. Med. Mycol. 47, 387-397. doi: 10.1080/ 13693780802609604

Prigitano, A., Esposto, M. C., Biffi, A., Lorenzis, G. D., Favuzzi, V., Koncan, R., et al. (2017). Triazole resistance in Aspergillus fumigatus isolates from patients with cystic fibrosis in Italy. J. Cyst. Fibros. 16, 64-69. doi: 10.1016/ j.jcf.2016.06.006

Risum, M., Hare, R. K., Gertsen, J. B., Kristensen, L., Johansen, H. K., HelwegLarsen, J., et al. (2020). Azole-Resistant Aspergillus fumigatus Among Danish Cystic Fibrosis Patients: Increasing Prevalence and Dominance of TR34/L98H. Front. Microbiol. 11, 1850. doi: 10.3389/fmicb.2020.01850

Seufert, R., Sedlacek, L., Kahl, B., Hogardt, M., Hamprecht, A., Haase, G., et al. (2018). Prevalence and characterization of azole-resistant Aspergillus fumigatus in patients with cystic fibrosis: a prospective multicentre study in Germany. J. Antimicrob. Chemother. 73, 2047-2053. doi: 10.1093/jac/dky147

Stevens, D. A., Moss, R. B., Kurup, V. P., Knutsen, A. P., Greenberger, P., Judson, M. A., et al. (2003). Allergic bronchopulmonary aspergillosis in cystic fibrosis - state of the art: Cystic Fibrosis Foundation Consensus Conference. Clin. Infect. Dis. 37, S225-S264. doi: 10.1086/376525

Ullmann, A. J., Aguado, J. M., Arikan-Akdagli, S., Denning, D. W., Groll, A. H., Lagrou, K., et al. (2018). Diagnosis and management of Aspergillus diseases: executive summary of the 2017 ESCMID-ECMM-ERS guideline. Clin. Microbiol. Infect. 24, e1-e38. doi: 10.1016/j.cmi.2018.01.002

Verweij, P. E., Ananda-Rajah, M., Andes, D., Arendrup, M. C., Brüggemann, R. J., Chowdhary, A., et al. (2015). International expert opinion on the management of infection caused by azole-resistant Aspergillus fumigatus. Drug Resist. Updat. 21-22, 30-40. doi: 10.1016/j.drup.2015.08.001

Wiederhold, N. P., and Verweij, P. E. (2020). Aspergillus fumigatus and pan-azole resistance: who should be concerned? Curr. Opin. Infect. Dis. 33, 290-297. doi: 10.1097/QCO.0000000000000662

Conflict of Interest: J-PG received research grants from Gilead, MSD, and Pfizer.

The remaining authors declare that the research was conducted in the absence of any commercial or financial relationships that could be construed as a potential conflict of interest.

Copyright (c) 2021 Guegan, Prat, Robert-Gangneux and Gangneux. This is an openaccess article distributed under the terms of the Creative Commons Attribution License (CC BY). The use, distribution or reproduction in other forums is permitted, provided the original author(s) and the copyright owner(s) are credited and that the original publication in this journal is cited, in accordance with accepted academic practice. No use, distribution or reproduction is permitted which does not comply with these terms. 